The Astrophysical Journal SUPPLEMEnt Series, 90:583-588, 1994 February

(C) 1994. The American Astronomical Socicty. All rights reserved. Printed in U.S.A.

\title{
ACCELERATION OF ELECTRONS AT A CURVED SHOCK
}

\author{
M. VANDAS \\ Astronomical Institute, Academy of Sciences, Boční II 1401/19, 14131 Praha 4, Czech Republic \\ Received 1993 February 10; accepted 1993 May 10
}

\begin{abstract}
The paper deals with the acceleration of electrons at a nearly perpendicular curved shock wave and results are compared with the case of a plane shock wave. We formulate the equations of motion of an electron in adiabatic approximation and solve them in a special case. The results show that the energy gain of reflected electrons at a curved shock depends on the ratio of the radius of curvature to the shock thickness; for a low ratio the energy gain is significantly lower than at a plane shock, while the energy gain of transmitted electrons is approximately the same as at a plane shock. The analytical solution provides a tool for direct calculations of fluxes of accelerated electrons near the curved shock of given parameters.
\end{abstract}

Subject headings: acceleration of particles - shock waves

\section{INTRODUCTION}

It is well-known that electrons at a nearly perpendicular shock are accelerated by the shock drift mechanism and this process is well understood in the case of a plane shock wave (Wu 1984; Leroy \& Mangeney 1984; Vandas 1989). For the acceleration to be efficient a nearly perpendicular shock wave is necessary because of high electron velocities, i.e., the angle $\theta_{B n}$ between the upstream magnetic field and the shock normal must be close to $90^{\circ}$ (within several degrees). The energy gain of electrons is very sensitive to $\theta_{B n}$ so the curvature of the shock (always present in real shocks) will play a very important and limiting role.

The present paper is a study and intercomparison of acceleration of electrons by a nearly perpendicular plane (one-dimensional case) and curved (two- and three-dimensional cases) shock waves. Krauss-Varban \& Burgess (1991) firstly dealt with a curved shock in the electron acceleration problem. They used a simulated shock and calculated numerically the motion of electrons in it for the two-dimensional case. As a result they presented expected fluxes of accelerated electrons near the curved shock. Our approach differs in two ways: (1) we study separately a single interaction of an electron with the curved shock trying to understand the dependence of the electron energy gain on initial quantities and shock parameters, and (2) we prefer analytical approach to this problem as far as it is possible. We treat the acceleration of suprathermal electrons, i.e., with energies hundreds and thousands of $\mathrm{eV}$.

\section{MODELS OF A SHOCK WAVE}

\subsection{One-Dimensional Case (a Plane Shock)}

The model (Vandas 1989) is presented in Figure 1. The upstream plasma velocity is $V_{1}=\left(V_{1}, 0,0\right)$, and magnetic field $\boldsymbol{B}_{1}=\left(B_{1} \cos \theta_{B n}, 0,-B_{1}\right)$. The precise value of $B_{1 z}$ should be $-B_{1} \sin \theta_{B n}$ but, for simplicity, we put $\sin \theta_{B n} \approx 1$ and $\cos \theta_{B n} \approx 0$ in expressions where it is possible because of $\theta_{B n} \approx 90^{\circ}$. The magnetic and electric fields inside the shock layer with the thickness $d$ are

$$
\boldsymbol{B}=\left(B_{1} \cos \theta_{B n}, 0,-B_{1} q\right), \quad \boldsymbol{E}=\left(0,-V_{1} B_{1}, 0\right),
$$

where $q(x)$ is an increasing function of $x$ from 1 to $\nu$ ( $\nu$ is the shock magnetic field jump) and defines the magnetic field profile in the shock. The electric field is constant everywhere and the magnetic field downstream is $B_{2}=\left(B_{1} \cos \theta_{B n}, 0,-B_{1} \nu\right)$.

We have chosen three types of magnetic field profiles for calculations:

$$
q(x)=\exp \left(\frac{x}{d} \ln \nu\right)
$$

(B) $q(x)=1+\frac{x}{d}(\nu-1)$,

$$
\text { (C) } q(x)=1+\tau\left[1-\exp (-\lambda x)\left(\cos \omega x+\frac{\lambda}{\omega} \sin \omega x\right)\right] \text {, }
$$

where $\omega=\pi / d, \lambda=1 / d$,

$$
\tau=\frac{\nu-1}{1+\exp (-1)}
$$

Case (A) fulfils the condition $q^{\prime} / q=$ constant $\left(q^{\prime}=d q / d x\right)$ important in an analytical treatment ( this is $\partial B / \partial s=$ constant in a very good approximation). Case $B$ is a linear profile and case $\mathrm{C}$ is similar to the observed magnetic field profiles (Vandas 1991a).

In our models we do not take into account electrostatic (barrier) field $\left(E_{x}\right)$ and noncoplanar magnetic field $\left(B_{y}\right)$ in the shock layer which are important mainly for thermal electrons.

\subsection{Two-Dimensional Case (a Curved Shock)}

The geometry of a curved shock in the two-dimensional case is presented in Figure 2 (Vandas 1991b). The shock wave has a cylindrical shape given by the equation $\left(R_{c}-x\right)^{2}+z^{2}=R_{c}^{2}$, where $R_{c}$ is the radius of curvature. The upstream plasma velocity is $V_{1}=\left(V_{1}, 0,0\right)$, and magnetic field $\boldsymbol{B}_{1}=\left(0,0,-B_{1}\right)$.

For a creation of a model of a curved shock, we have used an idea of Krauss-Varban \& Burgess (1991) to take a plane shock and modify its fields according to expected field dependences 


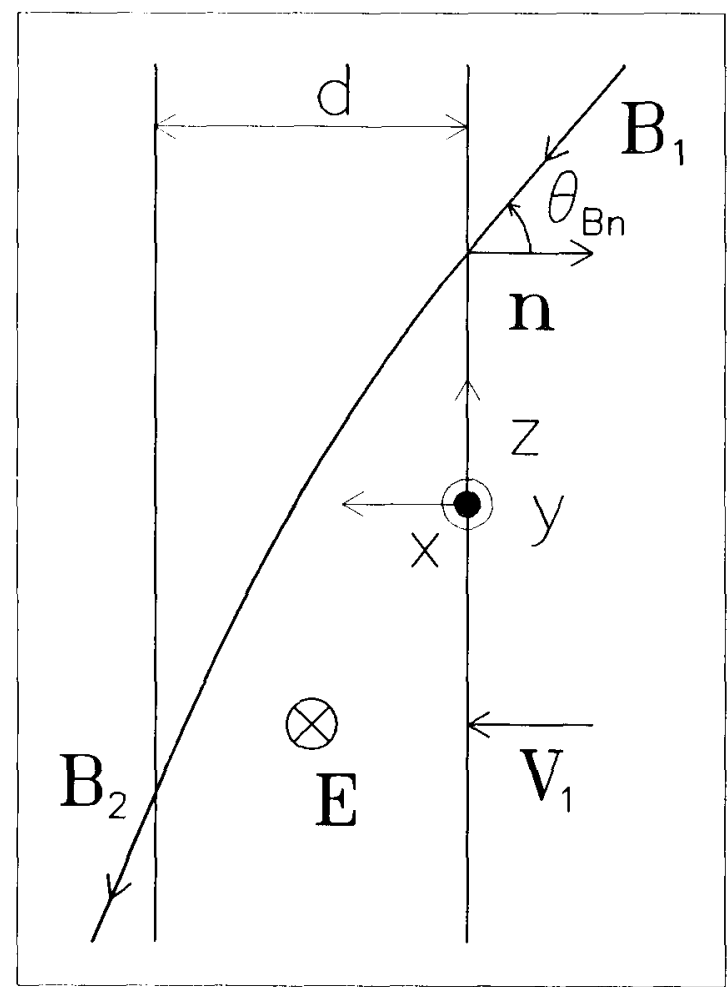

FIG. 1.-Plane shock with the thickness $d$ and the coordinate system definition: the shock is parallel to $y z$-plane. In the upstream region $(x<0)$ the plasma velocity is $V_{1}$ and magnetic field $\boldsymbol{B}_{1} . \boldsymbol{n}$ is the shock normal; it makes the angle $\theta_{B n}$ with $B_{1}, B_{2}$ is the magnetic field in the downstream region $(x>d)$, the thicker line is one of the magnetic field lines, $\boldsymbol{E}$ is the (motional) electric field.

on the varying angle $\theta_{B n}$. It follows from Figure 2 that the normal magnetic field component at the upstream side varies as $B_{n}=\left|B_{1} \cdot \boldsymbol{n}\right|=B_{1}\left(z / R_{c}\right)$. Thus we take for a model of a curved shock in two-dimensions the same geometry as in Figure 1 (i.e., a plane shock) but the magnetic and electric fields inside the shock layer will be

$$
\boldsymbol{B}=\left(B_{1} \frac{z}{R_{c}}, 0,-B_{1} q\right), \quad \boldsymbol{E}=\left(0,-V_{1} B_{1}, 0\right)
$$

The quantity $q(x)$ is defined as in the one-dimensional case.

\subsection{Three-Dimensional Case (a Curved Shock)}

The geometry of a curved shock in three-dimensional case is also as in Figure 2 (Vandas 1991b). The shock wave has a spherical shape given by the equation

$$
\left(R_{c}-x\right)^{2}+y^{2}+z^{2}=R_{c}^{2},
$$

where $R_{c}$ is the radius of curvature.

To create a model of a curved shock in three dimensions, we use the same idea as in the two-dimensional case. It follows from Figure 2 that the normal magnetic field component at the upstream side varies as $B_{n}=\left|B_{1} \cdot \boldsymbol{n}\right|=B_{1}\left(z / R_{c}\right)$, i.e., as in two-dimensional case. For the acceleration the normal compo- nent of the upstream velocity is in play,

$$
V_{1 n}=\left|V_{1} \cdot \boldsymbol{n}\right|=V_{1} \frac{R_{c}-x}{R_{c}}=V_{1} \sqrt{1-\left(\frac{y}{R_{c}}\right)^{2}}
$$

Thus we take for a model of a curved shock in three dimensions the same geometry as in Figure 1 (i.e., a plane shock) but the magnetic and electric fields inside the shock layer will be

$$
\begin{aligned}
\boldsymbol{B} & =\left(B_{1} \frac{z}{R_{c}}, 0,-B_{1} q\right), \\
\boldsymbol{E} & =\left[0,-V_{1} B_{1} \sqrt{1-\left(\frac{y}{R_{c}}\right)^{2}}, 0\right] .
\end{aligned}
$$

The quantity $q(x)$ is defined as in the one-dimensional case.

\section{STUDY OF THE ACCELERATION OF ELECTRONS}

Suprathermal electrons have a small gyroradius and we can use adiabatic approximation. In the following paragraphs we shall give equations of motion suitable for numerical calculations. In a special case an analytical solution of simplified equations of motion can be obtained.

\subsection{Results in the One-Dimensional Case (a Plane Shock)}

The case of a plane shock is well understood (Wu 1984; Leroy \& Mangeney 1984; Vandas 1989). The equations of

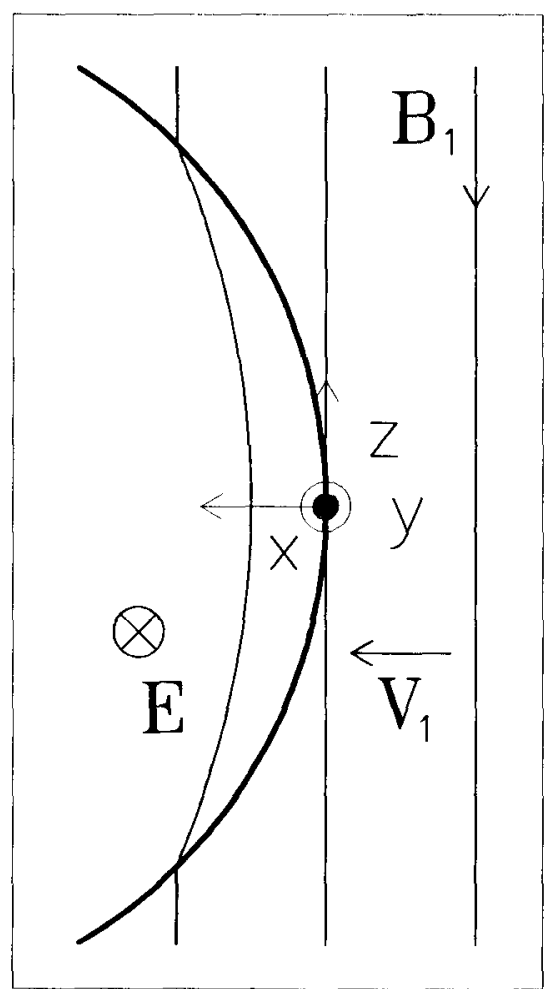

FIG. 2.-Geometry of a curved shock (thick line) and the coordinate system definition. In the upstream region ( right) the plasma velocity is $V_{1}$ and magnetic field $\boldsymbol{B}_{1}$. Three magnetic field lines are shown; $\boldsymbol{E}$ is the (motional) electric field. The angle $\theta_{B n}$ varies along the shock surface, it decreases from $90^{\circ}$ at the nose of the shock (the coordinates' origin). 
motion for the model in Figure 1 in adiabatic approximation are

$$
\begin{aligned}
\dot{v}_{\|} & =-\frac{\mu}{m} B_{1} \cos \theta_{B n} \frac{q^{\prime}}{q}-\frac{V_{1} q^{\prime} \cos \theta_{B n}}{q^{4}}\left(v_{\|} \cos \theta_{B n}+V_{1}\right) \\
& \approx-\frac{\mu}{m} B_{1} \cos \theta_{B n} \frac{q^{\prime}}{q}, \\
\dot{x} & =\frac{v_{\|}}{q} \cos \theta_{B n}+\frac{V_{1}}{q}, \\
\dot{y} & =\frac{\mu}{e} \frac{q^{\prime}}{q}-\frac{m}{e B_{1}} \frac{q^{\prime}}{q^{4}}\left(v_{\|} \cos \theta_{B n}+V_{1}\right)^{2} \approx \frac{\mu}{e} \frac{q^{\prime}}{q}, \\
\dot{z} & =-v_{\|}+\frac{V_{1}}{q^{2}} \cos \theta_{B n} \approx-v_{\|}, \\
\dot{E} & =e V_{1} B_{1} \dot{y} \approx \mu V_{1} B_{1} \frac{q^{\prime}}{q},
\end{aligned}
$$

where expressions on the right hand side after $\approx$ are a very good approximation, $E$ is the electron energy, $v_{\|}$is the electron velocity along the magnetic field, $\mu$ the magnetic moment, $e$ is the elementary charge, $m$ the electron mass. The initial values for the time $t=0$ are $v_{\|}=v_{i} \cos \alpha_{i}, x=0, y=y_{i}, z=z_{i}, E=E_{i}$. The quantity $v_{i}$ is the initial electron velocity, $E_{i}=\frac{1}{2} m v_{i}^{2}$ is the initial energy, $\alpha_{i}$ the initial pitch angle $\left(\alpha_{i}<90^{\circ}\right.$ is toward the shock), and

$$
\mu=\frac{m v_{1}^{2} \sin ^{2} \alpha_{i}}{2 B_{1}}
$$

The approximate equations can be solved for $q$ of case A:

$$
\begin{aligned}
& x=\frac{d}{\ln \nu} \ln \left\{\frac{\ln \nu}{d}\left[V_{1} t-\left(z-z_{i}\right) \cos \theta_{B n}\right]+1\right\}, \\
& y=\frac{\mu}{e} \frac{\ln \nu}{d} t+y_{i} \\
& z=\frac{1}{2} K_{1} t^{2}-v_{i} t \cos \alpha_{i}+z_{i} \\
& E=\mu V_{1} B_{1} \frac{\ln \nu}{d} t+E_{i}
\end{aligned}
$$

where

$$
K_{1}=\frac{\mu B_{1} \cos \theta_{B n} \ln \nu}{m d}
$$

If an electron crosses $x=d$ it is called transmitted, else if it returns to $x=0$ it is called reflected. Solving the equation $x=0$ we can obtain the interaction time $t_{\text {int }}$ of reflected electrons

$$
t_{\text {int }}=\frac{2}{K_{1}}\left(v_{i} \cos \alpha_{i}+\frac{V_{1}}{\cos \theta_{B n}}\right)=\frac{2}{K_{1}}\left(v_{i} \cos \alpha_{i}+V_{B}\right),
$$

and the known expression (Wu 1984; Leroy \& Mangeney
1984; Vandas 1989) for final energy $E_{f}$ of reflected electrons

$$
E_{f}=E_{i}+2 m V_{B}^{2}+2 m V_{B} v_{i} \cos \alpha_{i},
$$

where $V_{B}=V_{1} / \cos \theta_{B n}$ (a magnetic mirror velocity). In order for the acceleration of electrons to be efficient the velocity $V_{B}$ must be comparable to electron velocities, i.e., $\theta_{B n}$ close to $90^{\circ}$. Analogically for transmitted electrons we can get $t_{\text {int }}$ and $E_{f}$ solving the equation $x=d$.

It can be shown (Vandas 1989) that the expressions for $E_{f}$ are more general and $E_{f}$ does not depend on the shock structure $(q)$ and thickness $(d)$. The same is true for $\Delta y$, the total shift in $y$ in the shock layer $\left(\Delta y=y_{f}-y_{i}\right)$. The interaction time $t_{\text {int }}$ and shift $\Delta z$ do depend on the shock structure. The final pitch angle follows from the magnetic moment conservation.

For electrons breaking the adiabatic approximation $\left(r_{g} \approx d\right.$, $r_{g} \gg d$, where $r_{g}$ is the gyroradius, or even for $d=0$ ), direct numerical calculations of the equation of motion for an electron $m \ddot{\boldsymbol{r}}=-e(\boldsymbol{E}+\dot{\boldsymbol{r}} \times \boldsymbol{B})$ in the fields of the model show that the initial and final magnetic moments approximately coincide (but $\mu$ varies during the interaction). Also the final energy $E_{f}$, final pitch angle $\alpha_{f}$, and shift $\Delta y$ correspond to the results in adiabatic approximation (Vandas 1991a).

\subsection{Results in the Two-Dimensional Case (a Curved Shock)}

The equations of motion are

$$
\begin{aligned}
\dot{v}_{\|}= & -\frac{\mu}{m} B_{1} \frac{z}{R_{c}}\left(\frac{q^{\prime}}{q}-\frac{1}{R_{c} q}\right) \\
& -\frac{V_{1}}{q^{4}}\left[q^{\prime} \frac{z}{R_{c}}\left(v_{\|} \frac{z}{R_{c}}+V_{1}\right)+\frac{1}{R_{c}}\left(v_{\|} q^{2}-V_{1} \frac{z}{R_{c}}\right)\right] \\
\approx & -\frac{\mu}{m} B_{1} \frac{z}{R_{c}} \frac{q^{\prime}}{q}, \\
\dot{x}= & \frac{v_{\|}}{q} \frac{z}{R_{c}}+\frac{V_{1}}{q}, \\
\dot{y}= & \frac{\mu}{e}\left(\frac{q^{\prime}}{q}+\frac{z^{2}}{R_{c}^{3} q^{3}}\right) \\
& -\frac{m}{e B_{1} q^{4}}\left[q^{\prime}\left(v_{\|} \frac{z}{R_{c}}+V_{1}\right)^{2}+\frac{1}{R_{c}}\left(v_{\|} q-\frac{V_{1}}{q} \frac{z}{R_{c}}\right)^{2}\right]
\end{aligned}
$$

$$
\approx \frac{\mu}{e} \frac{q^{\prime}}{q}
$$

$$
\dot{z}=-v_{\|}+\frac{V_{1}}{q^{2}} \frac{z}{R_{c}} \approx-v_{\|}
$$

$\dot{E}=e V_{1} B_{1} \dot{y} \approx \mu V_{1} B_{1} \frac{q^{\prime}}{q}$

The initial values for the time $t=0$ are $v_{\|}=v_{i} \cos \alpha_{i}, x=0, y=$ $y_{i}, z=z_{i}=R_{c} \cos \theta_{B n i}, E=E_{i}$. 
The approximate equations can be solved for $q$ of case A:

$$
\begin{aligned}
& x=\frac{d}{\ln \nu} \ln \left\{\frac{\ln \nu}{d}\left[V_{1} t-\frac{1}{2 R_{c}}\left(z^{2}-z_{i}^{2}\right)\right]+1\right\} \\
& y=\frac{\mu}{e} \frac{\ln \nu}{d} t+y_{i} \\
& z=z_{i} \cosh K_{2} t-\frac{v_{i} \cos \alpha_{i}}{K_{2}} \sinh K_{2} t \\
& E=\mu V_{1} B_{1} \frac{\ln \nu}{d} t+E_{i}
\end{aligned}
$$

where

$$
K_{2}=\sqrt{\frac{\mu B_{1} \ln \nu}{m d R_{c}}}
$$

If an electron crosses $x=d$ it is called transmitted, else if it returns to $x=0$ it is called reflected (electrons with opposite signs of $z_{i}$ and $z_{f}$ are reflected through the nose). Setting $x=0$ we obtain an equation for the interaction time $t_{\text {int }}$ of reflected electrons

$$
\begin{aligned}
\left(z_{i} \cosh K_{2} t_{\mathrm{int}}-\frac{v_{i} \cos \alpha_{i}}{K_{2}} \sinh K_{2} t_{\mathrm{int}}\right)^{2} & \\
& -z_{i}^{2}-2 R_{c} V_{1} t_{\mathrm{int}}=0
\end{aligned}
$$

$\left(z_{i}=R_{c} \cos \theta_{B n i}\right)$. Opposite to a plane shock this is a transcendental equation and $t_{\text {int }}$ can be solved only numerically. Hence the final energy $E_{f}$ of reflected electrons

$$
E_{f}=E_{i}+\mu V_{1} B_{1} \frac{\ln \nu}{d} t_{\mathrm{int}}
$$

cannot be explicitly given. Nevertheless the numerical solution of $t_{\text {int }}$ is easy. Analogically for transmitted electrons we can get $t_{\text {int }}$ and $E_{f}$ solving the equation $x=d$.

The time dependence of the energy of interacting electrons practically does not depend on the curvature; it is the same as at a plane shock (cf. $\dot{E}$ in eqs. [5] and [9], see Fig. 3). But the interaction time is significantly lower for reflected electrons at a curved shock so the energy gain is correspondingly lower (Figs. 4 and 5). For a given thickness, a higher curvature gives a lower energy gain, in principle. Opposite to a plane shock case the energy gain of reflected electrons depends on the shock structure $(q)$ and namely on the thickness $(d)$ of the shock layer (Fig. 6). The decrease of thickness causes the increase in energy gain. In the limit $d \rightarrow 0$ (in fact unrealistic because adiabatic approximation is not valid) the solution gives $E_{f}$ as at a plane shock. In fact, direct numerical calculations using the Lorenz force for case $d=0$ give lower values of $E_{f}$.

A more detailed inspection of the equations (11) and (12) reveals that the final energy $E_{f}$ of reflected electrons depends on the ratio of the radius of curvature to the shock thickness $R_{c} / d$, and not on $R_{c}$ and $d$ separately. Figure 7 gives a rough

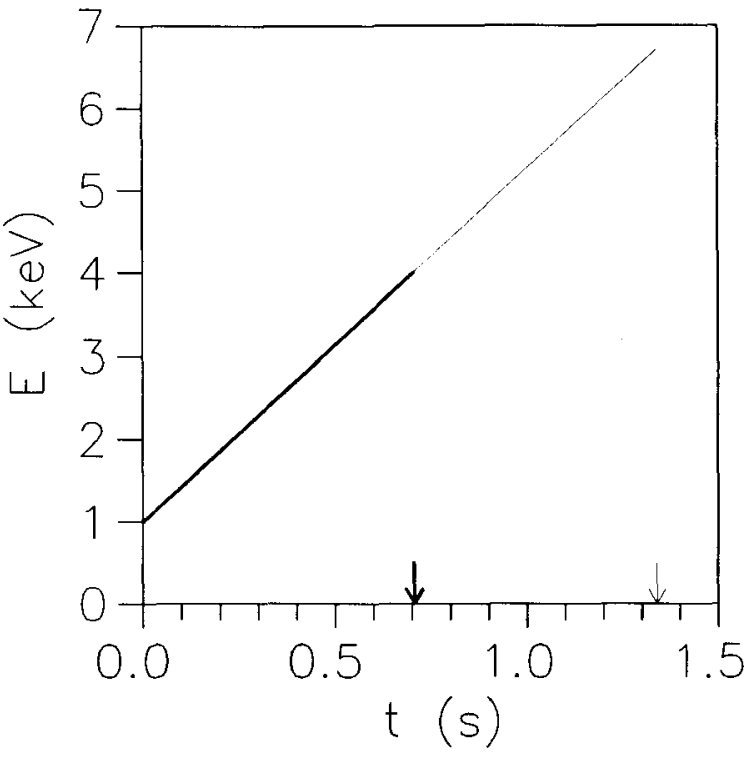

FIG. 3.-Time dependence of the energy $E$ of a reflected electron ( $t$ hick line) with the initial energy $E_{i}=1 \mathrm{keV}$, pitch angle $\alpha_{i}=80^{\circ}, \theta_{B n i}=88^{\circ} .9$ in the curved shock with the parameters $B_{1}=10 \mathrm{nT}, V_{1}=400 \mathrm{~km} \mathrm{~s}^{-1}, \nu=3$, $R_{c}=40 R_{E}, d=100 \mathrm{~km}, q$ of case A, in adiabatic approximation. The thin line shows the same for the plane shock with $\theta_{B n}=\theta_{B n i}$. Arrows indicate the exit time from the shock layer, i.e., the interaction time $t_{\text {int }}$ which is significantly lower for the curved shock.

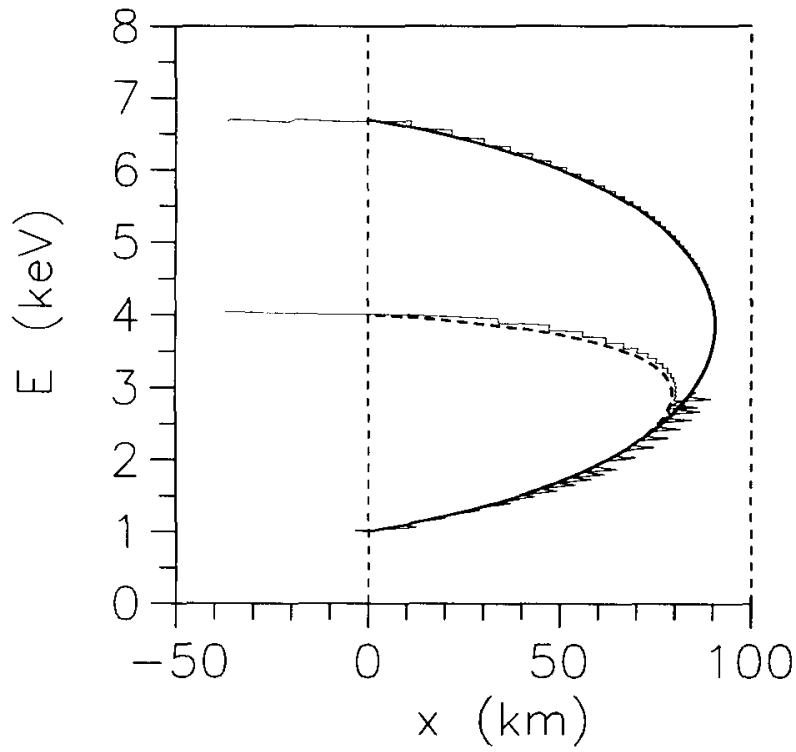

FIG. 4.-Change in energy $E$ of a reflected electron with the initial energy $E_{i}=1 \mathrm{keV}$, pitch angle $\alpha_{i}=80^{\circ}, \theta_{B n i}=88^{\circ} .9$ during its motion in the curved shock with the parameters $B_{1}=10 \mathrm{nT}, V_{1}=400 \mathrm{~km} \mathrm{~s}^{-1}, \nu=3$, $R_{c}=40 R_{E}, d=100 \mathrm{~km}, q$ of case A, as a function of the $x$-coordinate: the dashed thick line is adiabatic approximation, the thin line is a direct numerical calculation using the Lorenz force. The shock layer is situated between vertical dashed lines. Also shown are the results for the plane shock with $\theta_{B n}=\theta_{B n i}$ (the full thick line is adiabatic approximation, the thin line is a direct numerical calculation using the Lorenz force). A very good coincidence is seen between the analytical solutions and direct numerical calculations. 


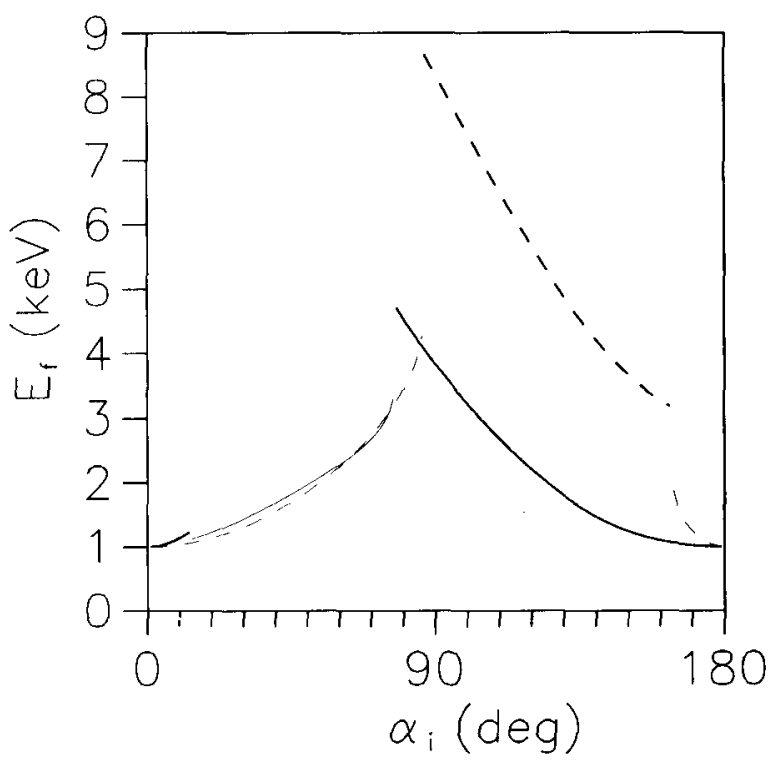

FIG. 5.-Dependence of the final energy $E_{f}$ of an electron with the initial energy $E_{i}=1 \mathrm{keV}, \theta_{B n i}=89^{\circ} 1$ on the pitch angle $\alpha_{i}$ (full line) at the curved shock with the parameters $B_{1}=10 \mathrm{nT}, V_{1}=400 \mathrm{~km} \mathrm{~s}^{-1}, \nu=3, R_{c}=$ $40 R_{E}, d=100 \mathrm{~km}, q$ of case $\mathrm{A}$, in adiabatic approximation. The thin line represents transmitted, the thick line reflected electrons. Dashed lines are results at the plane shock with $\theta_{B n}=\theta_{B n i}$. The plot illustrates that the energy gain of reflected electrons at a curved shock is significantly lower than at a plane one, and it is approximately the same for transmitted electrons. Opposite to a plane shock there are also reflected electrons for small $\alpha_{i}$ (reflected through the nose) and for $\alpha_{i}$ near $180^{\circ}$.

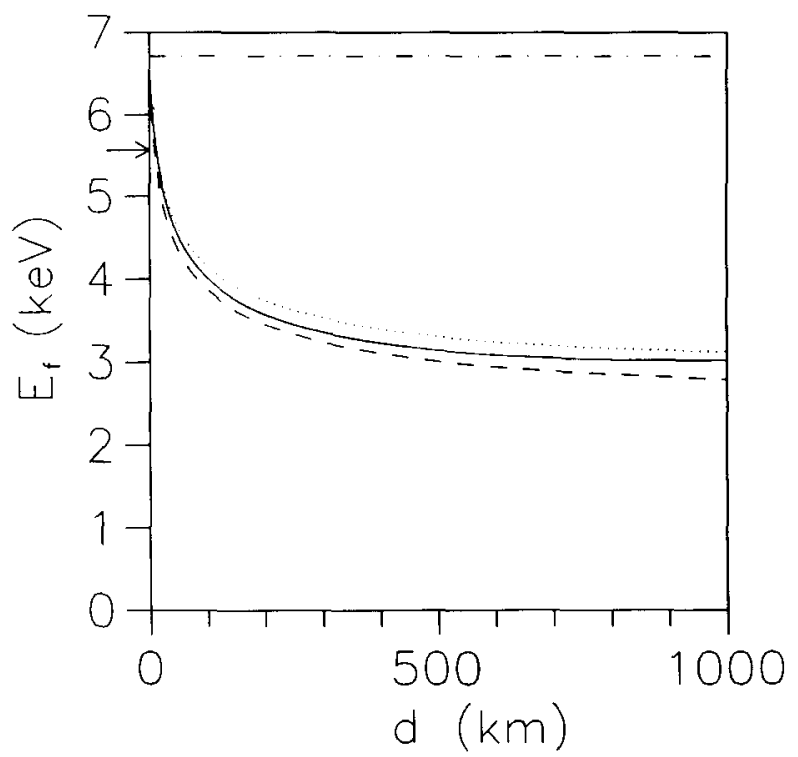

FIG. 6.-Dependence of the final energy $E_{f}$ of a reflected electron with the initial energy $E_{i}=1 \mathrm{keV}$, pitch angle $\alpha_{i}=80^{\circ}, \theta_{B n i}=88.9$ at the curved shock with the parameters $B_{1}=10 \mathrm{nT}, V_{1}=400 \mathrm{~km} \mathrm{~s}^{-1}, \nu=3, R_{c}=40 R_{E}$, on the shock thickness $d$ and magnetic field profile: full line for $q$ of case A, dashed line for $q$ of case B, dotted line for $q$ of case $\mathrm{C}$, in adiabatic approximation. The dashed-dotted line shows the same for the plane shock with $\theta_{B n}=\theta_{B n i}$ (no dependence on shock thickness and magnetic field profile). An arrow indicates the value of $E_{f}$ from direct numerical calculations using the Lorenz force for a model with $d=0$.

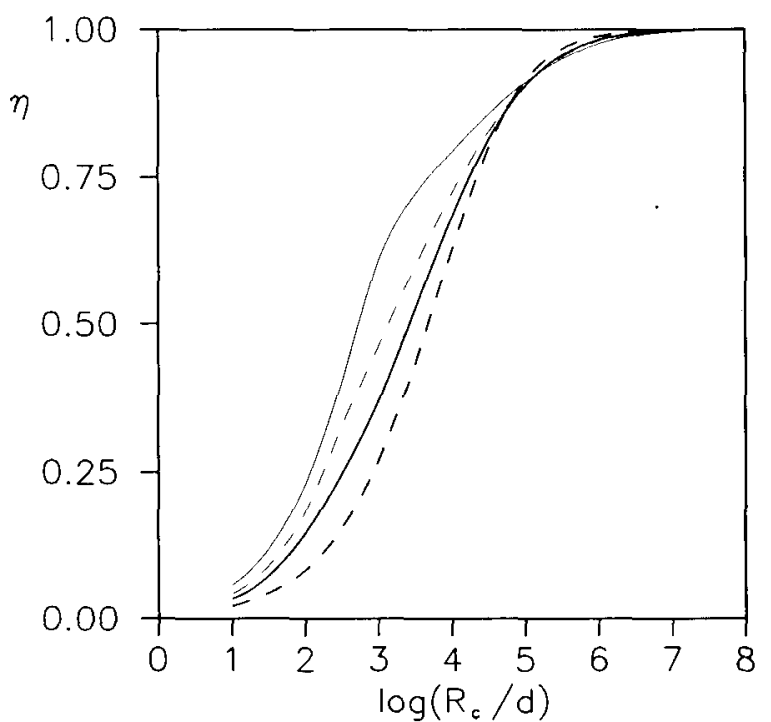

FiG. 7.-Dependence of the averaged energy gain $\eta$ of reflected electrons at a curved shock relative to a plane shock case on the ratio of the radius of curvature to the shock thickness $R_{c} / d$.

$$
\eta=\left\langle\frac{\left(E_{f}-E_{i}\right)_{c}}{\left(E_{f}-E_{i}\right)_{p}}\right\rangle,
$$

where the subscript $c$ is for a curved shock, and $p$ is for a corresponding plane shock. $\eta$ was averaged over initial pitch angles $\alpha_{i}$ and incident field angles $\theta_{B n i}$ in the interval $88^{\circ}-90^{\circ}$. We used the above given fraction in the definition of $\eta$ instead of $\left(E_{f}\right)_{c} /\left(E_{f}\right)_{p}$ because the former is less sensitive to the upstream plasma velocity $V_{1}$ (see eq. [12]). Shocks have the parameters $B_{1}=10 \mathrm{nT}, v=3, q$ of case A. The thicker line is for $E_{i}=1 \mathrm{keV}$, the thin line is for $E_{i}=10 \mathrm{keV}$. The full lines are for the upstream plasma velocity $V_{1}=400 \mathrm{~km} \mathrm{~s}^{-1}$, the dashed lines are for $V_{1}=800 \mathrm{~km} \mathrm{~s}^{-1}$.

imagination of the influence of curvature on the energy gain of reflected electrons. $\eta$ is the energy gain of reflected electrons at a curved shock relative to a plane shock averaged over initial pitch angles $\alpha_{i}$ and incident field angles $\theta_{B n i}$. In the range of the Earth's bow shock, i.e., $R_{c} / d-10^{2}-10^{3}$, we see a large influence of curvature; the energy gain is about $\frac{1}{4}$ of a plane shock case. For interplanetary shocks, the ratio can be estimated to $10^{4}-10^{5}$ where the energy gain is about $\frac{3}{4}$ of a plane shock case.

In general, the energy gain of transmitted electrons is lower than for reflected ones. This can be explained by shorter interaction times: transmitted electrons move only forward in the shock layer while reflected electrons forward and backward (see Figs. 4 and 3). The energy gain of transmitted electrons is approximately the same as at a plane shock with the corresponding $\theta_{B n}=\theta_{B n i}$ (Fig. 5). This can be understood if we realize that the evolution in energy gain differs only slightly from a plane shock case during the forward part of the electron trajectory, but it differs very much during the backward part (see Fig. 4).

Electrons reflected through the nose are electrons with small initial pitch angles $\alpha_{i}$, and $\theta_{B n i}$ close to $90^{\circ}$ (within $1^{\circ}-2^{\circ}$ ) which in case of a plane shock wave are transmitted. Their energy gain is only slightly larger than that of transmitted electrons (Fig. 5).

The dependence if an electron will be reflected or transmitted is more complicated than in a plane shock case and it can 


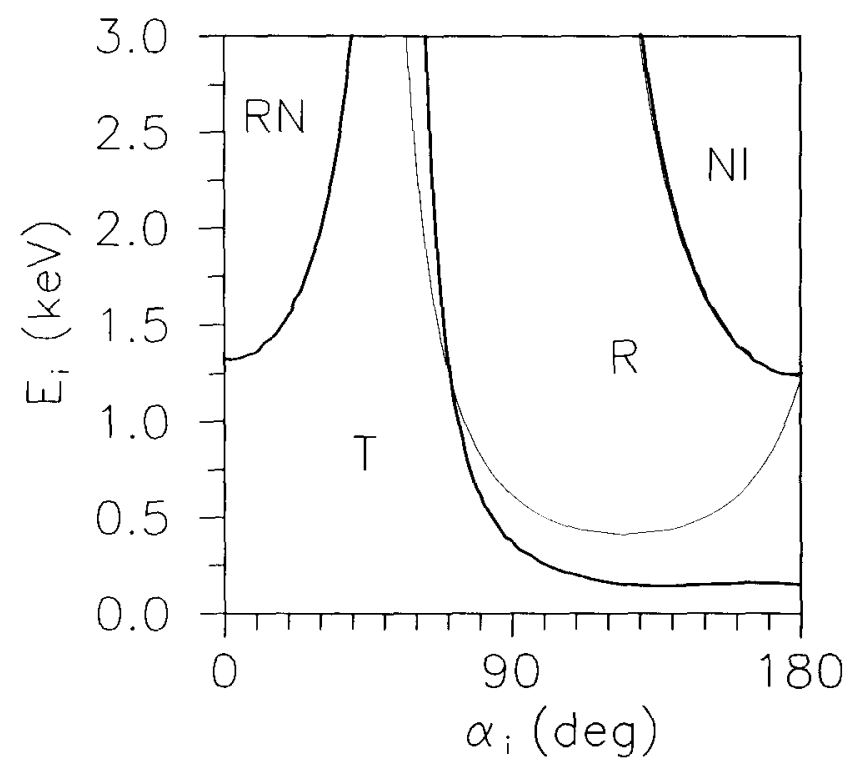

FIG. 8.-Plot maps a type of interaction of an electron with the initial energy $E_{i}$, pitch angle $\alpha_{i}$, and $\theta_{B n i}=88^{\circ} 9$ with the curved shock with the parameters $B_{1}=10 \mathrm{nT}, V_{1}=400 \mathrm{~km} \mathrm{~s}^{-1}, \nu=3, R_{c}=40 R_{E}, d=100 \mathrm{~km}, q$ of case $\mathrm{A}$, in adiabatic approximation. The thick curves divide the plot into four regions: $T$ are transmitted, $\mathbf{R}$ are reflected, NI are noninteracting electrons, and RN are electrons reflected through the nose. The thin curves (one coincides with the thick R/NI line) show a plane shock case with $\theta_{B n}=\theta_{B n i}$. In the latter case the region RN does not exist, it is a part of the region $\mathrm{T}$.

be evaluated only numerically (Fig. 8). Opposite to a plane shock, with increasing $\theta_{B n i}$ there are always present reflected electrons, and reflection or transmission depend on the shock thickness.

\subsection{Results in the Three-Dimensional Case (a Curved Shock)}

The equations of motion are the same as in the two-dimensional case if we make the substitution

$$
V_{1} \rightarrow V_{1} \sqrt{1-\frac{y^{2}}{R_{c}^{2}}}
$$

in equation (9). Because $y / R_{c} \approx y_{b} / R_{c}$, the three-dimensional case can be converted with good accuracy into the two-dimensional case if we only take the proper upstream plasma velocity in two-dimensional equations, i.e., make the change

$$
V_{1} \rightarrow V_{1} \sqrt{1-\frac{y_{i}^{2}}{R_{c}^{2}}}
$$

(also constant velocity) in equations (9)-(12).

\section{CONCLUSIONS}

1. The presented expressions enable calculation of electron trajectories and energy gains at a plane and curved shocks in adiabatic approximation and open way to calculate directly fluxes of electrons near a curved shock;

2. Energy gain of electrons at a curved shock is thickness and (slightly) structure dependent;

3. Energy gain of reflected electrons at a curved shock depends on the ratio of the radius of curvature to the shock thickness, it is an increasing function of this ratio with an upper limit given by the energy gain at a plane shock;

4. The influence of curvature on the energy gain of reflected electrons can be roughly estimated from Figure 7: the energy gain at the Earth's bow shock is significantly lower than at a plane shock;

5. Energy gain of transmitted electrons at a curved shock is approximately the same as at a plane shock;

6. Acceleration at a three-dimensional curved shock can be converted into the two-dimensional case.

Maximum energy gain of electrons reflected at a plane shock is $10 \times$ for $\nu=3$ and $18 \times$ for $\nu=5$, and it is lower for a curved shock. The equation for $E$ in equation (10) tells us that the energy gain depends significantly on the time spent in the shock layer $\left(t_{\text {int }}\right)$. If a process (like scattering) holds electrons longer in the shock layer, they could get considerably higher energies, mainly for shocks with a large extent and thickness (application to SNR? - but this should need a relativistic generalization ).

This work was supported by the grant of the Czechoslovak Academy of Sciences No. 30308 and the grant of the Academy of Sciences of the Czech Republic No. 303110.

\section{REFERENCES}

Krauss-Varban, D., \& Burgess, D. 1991, J. Geophys. Res., 96, 143 Leroy, M., \& Mangeney, A. 1984, Ann. Geophys., 2, 449 Vandas, M. 1989, Bull. Astron. Inst. Czechoslovakia, 40, 189
Vandas, M. 1991 a, Bull. Astron. Inst. Czechoslovakia, 42, 170 1991b, Proc. 22d Internat. Cosmic Ray Conf. (Dublin), 3, 280 Wu, C. 1984, J. Geophys. Res., 89, 8857 